\title{
An Integrated System for the Analysis and the Recognition of Characters in Ancient Documents
}

\author{
Stefano Vezzosi, Luigi Bedini, and Anna Tonazzini \\ Istituto di Elaborazione della Informazione - CNR \\ Via G. Moruzzi, 1, I-56124 PISA, Italy \\ \{vezzosi, bedini, tonazzini\}@iei.pi.cnr.it
}

\begin{abstract}
This paper describes an integrated system for processing and analyzing highly degraded ancient printed documents. For each page, the system reduces noise by wavelet-based filtering, extracts and segments the text lines into characters by a fast adaptive thresholding, and performs OCR by a feed-forward back-propagation multilayer neural network. The probability recognition is used as a discriminant parameter for determining the automatic activation of a feed-back process, leading back to a block for refining segmentation. This block acts only on the small portions of the text where the recognition was not trustable, and makes use of blind deconvolution and MRF-based segmentation techniques. The experimental results highlight the good performance of the whole system in the analysis of even strongly degraded texts.
\end{abstract}

\section{Introduction}

Standard Optical Character Recognition (OCR) systems, based on preliminary character segmentation, fail when applied to ancient printed texts, where aging of paper and diffusion of ink caused non-uniform contrast reduction of the images, presence of broken and touching characters, and background noise. Also Intelligent Character Recognition (ICR) systems, based on neural networks, though more robust against degradation, require correctly segmented characters. Often, the degradation can be modeled as an unknown space-variant blur plus additive noise, so that blind deconvolution is appropriate to enhance/restore the image. This highly underdetermined problem requires the adoption of a priori information and regularization strategies. Markov Random Fields (MRF) are efficient and flexible in modeling images, and allow for performing blind restoration and character segmentation jointly. To reduce the high computational complexity of these techniques, we use them for refining segmentation only on those zones of the document where the recognition fails. We thus define an integrated system that first applies a neural network classifier to a document page, preprocessed by simple and fast image enhancement and character segmentation techniques, and then, based on the recognition probability, automatically re-processes those small image areas where broken or touching characters were not correctly recognized. For small areas, blind restoration coupled with MRF segmentation is relatively cheap, allows for managing space-variant degradations, and is very effective in producing properly segmented, and then recognizable, characters.

D. Lopresti, J. Hu, and R. Kashi (Eds.): DAS 2002, LNCS 2423, pp. 49-52 2002.

(C) Springer-Verlag Berlin Heidelberg 2002 


\section{From the Document to the Characters}

We assume that the degraded image $\mathbf{g}$ of a document page can be modeled as $\mathbf{g}=H(d) \mathbf{f}+\mathbf{n}$, where $\mathbf{f}$ is the ideal image, $d$ is the blur mask, $H(d)$ is the matrix performing $2 D$ convolution, and $\mathbf{n}$ is a white Gaussian noise.

The integrated system for the analysis of the document is constituted of various processing blocks that interact with each other as shown in Fig. 1. The first

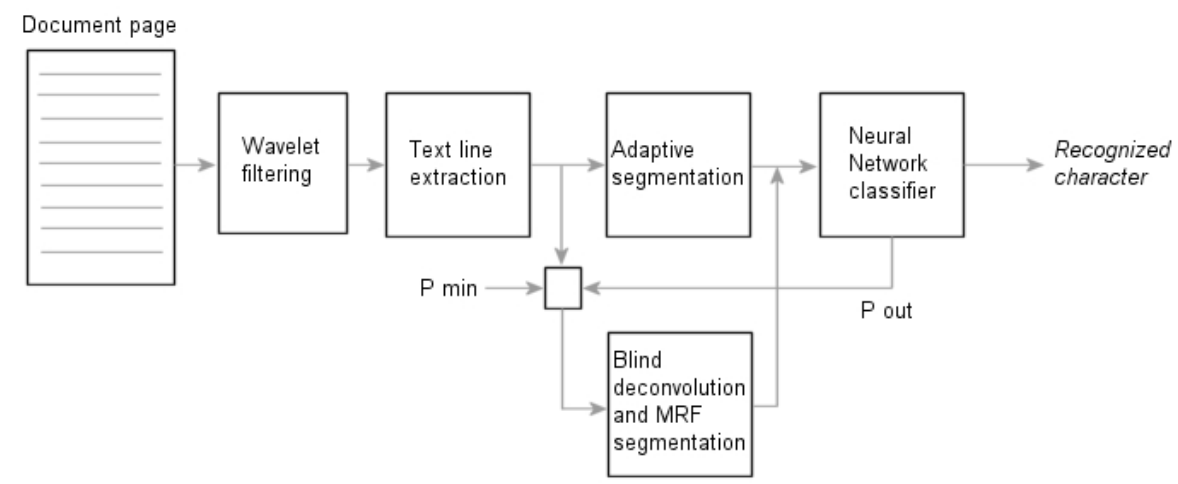

Fig. 1. Integrated system for document analysis and character recognition

block is designed to perform denoising through wavelet-based filtering. Neglecting the blur, the degraded image is decomposed by using the discrete wavelet transform (DWT), with the symlet function as the mother wavelet. In order to remove noise, the high frequency terms of the decomposition that are lower than the noise power are discarded via soft thresholding [1, and the others are used to reconstruct the image. This allows for preserving the character boundaries, usually mapped by frequency terms higher than the noise power.

The second step is devoted to the the extraction of the text lines. Since the lines are repeated in the page with an approximately fixed vertical distance, the projection of the page on the vertical axis is a periodic signal. The peak of the amplitude of the Fourier transform of this signal gives the repetition frequency of the text lines. Thus, once known the position of the first, all the lines can be extracted in sequence.

In the next step, each text line is segmented into blobs corresponding to the characters. The line is first converted into a two-level image, where the two levels, $a<b$, are the peaks of the bimodal hystogram, and correspond to the characters and the background, respectively. All pixels with value below a suitable threshold th are assigned with $a$ and the others with $b$. The blobs are then segmented by extracting the connected components at value $a$. Since the quality of the image greatly varies across the page, the threshold is adaptively computed on small 
portions of the text line, as the one that minimizes the distance between the gray level image and the corresponding binarized image.

In the fourth block of the system, the blobs of each line are individually and sequentially processed by a neural network classifier. We used a multilayer feedforward network, with a hidden layer, trained with an adaptive back-propagation algorithm, that uses the batch gradient descent with momentum and variable learning rate, to avoid local minima 2]. The training set was constituted of characters selected from a page of the document, and previously binarized and segmented with the techniques described above. We found that when the recognition percentage is above $90 \%$, the recognition is correct and the corresponding segmentation is accurate. Below this value, both the recognition and the segmentation are usually wrong. In these cases, the corresponding sub-image is re-processed by taking the degradation into account. The new segmented blobs are given again as input to the network. If the percentage is now higher than $90 \%$, the recognition is accepted, otherwise the blob is classified as unrecognizable.

\section{Segmentation Refining Procedure}

The refining of the segmentation is performed by the fifth processing block. When two adjacent blobs, recognized with low probabilities, are both smaller than a minimum size, they are joined into a single blob. When the recognition probability is low and the blob is large it likely corresponds to two or more joined characters. To split the characters we perform blind deconvolution [3] coupled with MRF image models [4], designed to produce a binarized solution. We define an energy function constituted of a data coherence term, plus a term related to the MRF model chosen. It is:

$$
E(\mathbf{f}, d)=\|\mathbf{g}-H(d) \mathbf{f}\|^{2}+\lambda U(\mathbf{f})
$$

where $U(\mathbf{f})$ is the prior MRF energy, and $\lambda$ is the regularization parameter. $U(\mathbf{f})$ describes constraints on the geometry of local configurations of the pixels. We adopted an extension of the Ising model to enforce smoothness on pairs of adjacent pixels [4, and to describe peculiar configurations of text pixels [5]. Since $E(\mathbf{f}, d)$ is non-convex, and has mixed, continuous and discrete, variables, we minimize it via the following iterative alternate scheme [6]:

$$
\begin{aligned}
d^{k} & =\operatorname{argmin}_{d}\left\|\mathbf{g}-H(d) \mathbf{f}^{k}\right\|^{2} \\
\mathbf{f}^{(k+1)} & =\operatorname{argmin}_{\mathbf{f}}\left\|\mathbf{g}-H\left(d^{(k)}\right) \mathbf{f}\right\|^{2}+\lambda U(\mathbf{f})
\end{aligned}
$$

Within a simulated annealing (SA) scheme governed by a decreasing temperature parameter [7], (2) is solved via least mean squares, and a Metropolis algorithm [7] is used to update $\mathbf{f}$ in (3). This approach is very efficient for refining segmentation, thus improving recognition as well. Its high computational complexity does not significantly affect the cost of the overall system, since it is applied to a few, small areas of the page. Moreover, the back-forward loop is governed by simple parameters, i.e. the minimum probability for accepting the recognition of a character, and the minimum and maximum extent allowed for the blobs. 


\section{Discussion of the Experimental Results}

The integrated system has been tested on some pages of the Opera Omnia by Girolamo Cardano. Here we provide the results of the analysis of one of the text lines. Fig. 2 shows the line segmented into blobs through the adaptive thresholding algorithm. Though satisfactory, the segmentation presents some

\section{principales Robur virium,Morbi exiften-}

Fig. 2. Segmentation superposed to the filtered gray level image

errors: the two " $\mathrm{n}$ " are both broken into two separated blobs, and the adjacent "o" and "b" are joined into a single blob. These errors are detected by the neural network, that gives a recognition probability lower than $90 \%$ for the blobs. These are back-forwarded to the segmentation refining procedure. For the " $n$ ", the two small blobs were connected into a single one. For the large blob "ob", we employed 15 iterations of scheme (2-3), obtaining a correct separation (Fig. $3)$. The final recognition resulted in the string "principales (t)obur virium(l)

\section{principales Robur virium, Morbi exiften-}

Fig. 3. Refined segmentation superposed to the filtered gray level image.

(si)orbi existen -", where only three blobs, over a total of 34 , are recognized with a probability lower than $90 \%$. We highlight that the recognition errors are due to the presence, in the training set, of only a few samples of characters $\mathrm{R}, \mathrm{M}$ and comma.

\section{References}

1. Donoho, D.L.: IEEE Trans. Information Theory. 41 (1995) 613-627.

2. Vogl, T.P. et al.: Biological Cybernetics. 59 (1988) 256-264.

3. Kundur, D., Hatzinakos, D.: IEEE Sig. Proc. Mag. (1996) 43-62.

4. Li, S.Z.: Markov Random Field Modeling in Computer Vision. (1995) SpringerVerlag Tokyo.

5. Tonazzini, A., Bedini, L.: Proc. 10th ICIAP. (1999) 836-841.

6. Ayers, G.R., Dainty, J.G.: Opt. Lett. 13 (1988) 547-549.

7. Aarts, E., Korst, J.: Simulated Annealing and Boltzmann Machines. (1989) Wiley. 